\title{
Joint Beamforming and Handoff for Traffic Improvements in Wireless Networks
}

\author{
Javad Razavilar, ${ }^{1}$ Farrokh Rashid-Farrokhi, ${ }^{2}$ and K.J. Ray Liu ${ }^{3}$ \\ ${ }^{1} 3 \mathrm{COM}$ Labs, 3COM Inc. \\ 12230 World Trade Drive \\ San Diego, CA 92128 \\ javad_razavilar@3com.com \\ ${ }^{2}$ Wireless Communications Research Dept. \\ Bell Labs, Lucent Technologies \\ 791 Holmdel-Keyport Rd, Holmdel, NJ 07733 \\ ${ }^{3}$ Electrical Engineering Department and Institute for Systems Research
University of Maryland
College Park, MD 20742
}

\begin{abstract}
A cellular wireless network with beamforming capabilities at the receiver is considered which allows two or more transmitters to share the same channel for communicating with the base station. A novel approach is introduced which combines the effects of the digital signal processing at the physical layer along with the network layer protocol on the overall queuing model of a cell. From this effective cell model, we find the closed form solutions for blocking probabilities of the calls and total carried traffic in the system. Our analytical as well as numerical results show that adaptive beamforming at the receiver reduces the blocking probability of the calls and increases the total carried traffic in the system.
\end{abstract}

Keywords: Antenna Arrays, Wireless Networks, Handoff, Adaptive Beamforming.

\section{Introduction}

In a cellular wireless communication network, as a mobile user crosses the boundaries of cells (coverage area of each base station) the call must be handed over to the new base station (BS) that provides the best link quality. This operation is called a handoff and ideally goes unnoticed by the customer [1]. Due to fixed finite resources, we cannot assume that more physical radio channels will be added to a spectrum allocation to reduce the probability of blocking or forced termination [1]. The reallocation of bandwidth is a standards- setting process that takes a considerably long time The increasing popularity of wireless communication services together with the limited amount of the available radio spectrum calls for highly efficient usage of resources (traffic channels) [2]. The interference reduction capability of antenna arrays have been considered as a means to increase the capacity of wireless systems [3, 4]. Specifically, using beamforming techniques at the receiver, two or more transmitters can share the same traffic channel to communicate with the base station at the same time. An adaptive antenna array is used at the base station to form several antenna beams simultaneously. Each beam captures one transmitter by automatically pointing its pattern toward that transmitter while nulling other cochannel transmitters [4]. In urban wireless environments several copies of the transmitted signal are received at the base station with different delays and different attenuation. This effect is called multipath fading. In a space only diversity, given a cell with $L$ channels, an $M$ element antenna array that forms $M-1$ distinct array nulls at the receiver allows a maximum of $K L$ users to be served in a cell, where $K=\frac{M}{\alpha}$ and $\alpha$ is the multipath factor representing the number of distinct strong path components received from each mobile station at the beamforming array. In a space-time diversity spatial nulls can be used to reject the cochannel interference (CCI), while inter-symbol interference (ISI) can be eliminated by time diversity. In this paper the queuing model of a network with joint beamforming and handoff under a given traffic policy is derived and from there the blocking probabilities of the calls and total carried traffic in the system are derived. 
The rest of the paper is organized as follows. A review of spatial multiplexing using adaptive beamforming techniques forms the subject of section 2. Network models with joint beamforming and handoff and the analysis of blocking probabilities under guard channel policy with or without queuing of handoff calls are presented in section 3. In section 4, the numerical results are provided. Finally we conclude the paper with section 5 where we summarize the obtained results.

\section{Review of Spatial Multiplexing Using Adaptive Beamforming}

A antenna array is a multibeam adaptive array with its gain pattern being adjusted dynamically [3]. An antenna array consisting of $M$ elements is considered at the receiver. A set of $J$ cochannels transmitters is considered. Let $s_{j}(k)$ for $j=1,2, \cdots, J$ denote the $j$ th transmitted signal. We assume that at most $N$ multipath signals frorn each user would arrive in the base station with different delays $\left(\tau_{n}\right)$. Therefore the received signal vector due to the $s_{i}(k)$ would be

$$
\mathbf{x}(k)=\sum_{j=1}^{J} \sqrt{P_{j} G_{j}} \sum_{n=1}^{N} \alpha_{j}^{n} \mathbf{a}\left(\theta_{j}^{n}\right) s_{j}\left(t-\tau_{n}\right)+\mathbf{n}(k)
$$

where $\theta_{i}^{n}$ is the arrival direction of the $n$th multipath signal from the $i$ th user, $P_{i}$ is the power of the $i$ th transmitter, $G_{i}$ is the link gain between the $i$ th transmitter and the base station, $\alpha_{i}^{n}$ is the $n$th path fading, $\mathbf{n}(k)$ is a zero mean Gaussian random vector with variance of each element $\sigma^{2}$, and $\mathbf{a}\left(\theta_{i}^{n}\right)$ is the array response to the multipath signal arriving from direction $\theta_{i}^{n}$ with $\mathbf{a}\left(\theta_{i}^{n}\right)=\left[a_{1}\left(\theta_{i}^{n}\right), a_{2}\left(\theta_{i}^{n}\right), \cdots, a_{M}\left(\theta_{i}^{n}\right)\right]^{T}$. In a space diversity combiner, illustrated in Figure 1 , a weighted sum of the outputs of the array elements is generated by a beamformer in the following way

$$
y_{i}(k)=\mathbf{w}_{i}^{H} \mathbf{x}(k),
$$

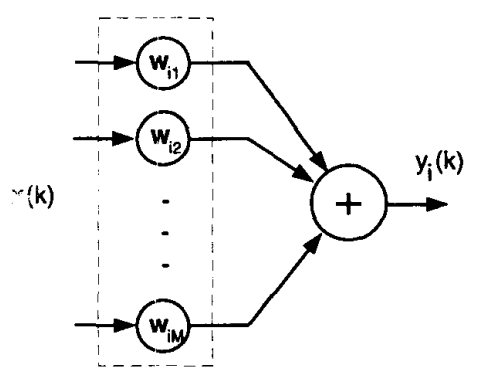

Figure 1: Space diversity combiner

where $\mathbf{w}_{i}^{T}=\left[w_{i 1}, \ldots, w_{i M}\right]$ is the weight vector and $\mathbf{x}^{T}(k)=\left[x_{1}(k), \ldots, x_{M}(k)\right]$ is the received signal vector. The optimum weight vector, in a minimum variance distortionless response (MVDR) beamformer
$[5,6]$, is the one that minimizes the interference while maintaining a unity gain at the direction of inter $\epsilon$ st by imposing $\mathbf{w}_{i}^{H} \mathbf{a}_{i}\left(\theta_{i}^{1}\right)=1$. The solution to this problem is [6]

$$
\hat{\mathbf{w}}_{i}=\frac{\Phi^{-1} \mathbf{a}_{i}\left(\theta_{i}^{1}\right)}{\mathbf{a}_{i}^{H}\left(\theta_{i}^{1}\right) \Phi^{-1} \mathbf{a}_{i}\left(\theta_{i}^{1}\right)}
$$

where

$$
\begin{aligned}
\mathbf{\Phi}= & P_{i} G_{i} \alpha_{i}^{1} \mathbf{a}_{i}\left(\theta_{i}^{1}\right) \mathbf{a}_{i}^{H}\left(\theta_{i}^{1}\right) \\
& +\sum_{j=1, j \neq i}^{J} P_{j} G_{j} \sum_{n=2}^{N} \alpha_{j}^{n} \mathbf{a}_{j}\left(\theta_{j}^{n}\right) \mathbf{a}_{j}^{H}\left(\theta_{j}^{n}\right)+\frac{N N_{0}}{2} I .
\end{aligned}
$$

The optimum weight vector maximizes the CIR at the output of beamformer, for the signal of interest, i.e.

$$
\begin{aligned}
\Gamma_{i, m a x}= & {\left[\sum_{j=1, j \neq i}^{J} P_{j} G_{j} \sum_{n=2}^{N} \alpha_{j}^{n} \hat{\mathbf{w}}_{i}^{H} \mathbf{a}_{j}\left(\theta_{j}^{n}\right) \mathbf{a}_{j}^{H}\left(g_{j}^{n}\right) \hat{\mathbf{w}}_{i}\right.} \\
& \left.+\frac{N_{0}}{2} \hat{\mathbf{w}}_{i}^{H} \hat{\mathbf{w}}_{i}\right]^{-1} P_{i} G_{i} .
\end{aligned}
$$

If the array response is not known, one can employ a training sequence and minimize the difference between the training sequence and the output of the jeamformer in mean square sense, i.e.

$$
\hat{\mathbf{w}}_{i}=\arg \min _{w_{i}} E\left[\left|d_{i}-\mathbf{w}_{i}^{H} \mathbf{a}_{i}\left(\theta_{i}^{1}\right)\right|^{2}\right]
$$

The solution to this problem is [6]

$$
\hat{\mathbf{w}}_{i}=\Phi_{i}^{-1} \mathbf{p}_{i}
$$

where $\Phi_{i}$ is defined as before and $\mathbf{p}_{i}$ is the cross correlation between the received vector and the training sequence; $\mathbf{p}_{i}=E\left[\mathbf{x}_{i} d_{i}^{*}\right]$.

\section{Network With Adaptive Array Under Guard Channels Traffic Policy}

In this section we analyze the network with adaptive array under guard channels traffic (GCT) policy proposed in [7]. The GCT policy is the blocking of newlyoriginating calls as soon as only $g$ channels among all available channels are still free [7]. This allows us to build a guard band before handoff calls will be blocked, which will happen only when all channels are occupied. In our network model, we assume that when a new call (hand off or originating call) arrives, the adaptive array points one beam toward that user and assigns one channel out of those $L$ channels to that user. Each channel $c_{i}$ for $i=1,2, \cdots, L$ can be assignec. to $K$ 
users by $K$ separate beams, using $K$ beamformers in parallel. If the first beam of all channels are already occupied by one user ( $L$ users are in the system) the new call can be assigned by another separate beam to any of the $L$ channels. If we assume that $(i-1)$ cochannel transmitters successfully share the same channel, for acceptable link quality, the newly arrived ith transmitter is allowed to share that channel with them if $\Gamma_{i} \geq \gamma$, where $\Gamma_{i}$ is given by (5), and $\gamma$ is a system parameter, dictated by the underlying standard governing the wireless network. To avoid any degradations in system performance, using a call admission control (CAC) mechanism, if $\Gamma_{i}<\gamma$ or $i>K$ we prevent that user from being accepted into the system. The flow chart of the channel assignment in a cell with an antenna array at the base station is shown in Figure 2.

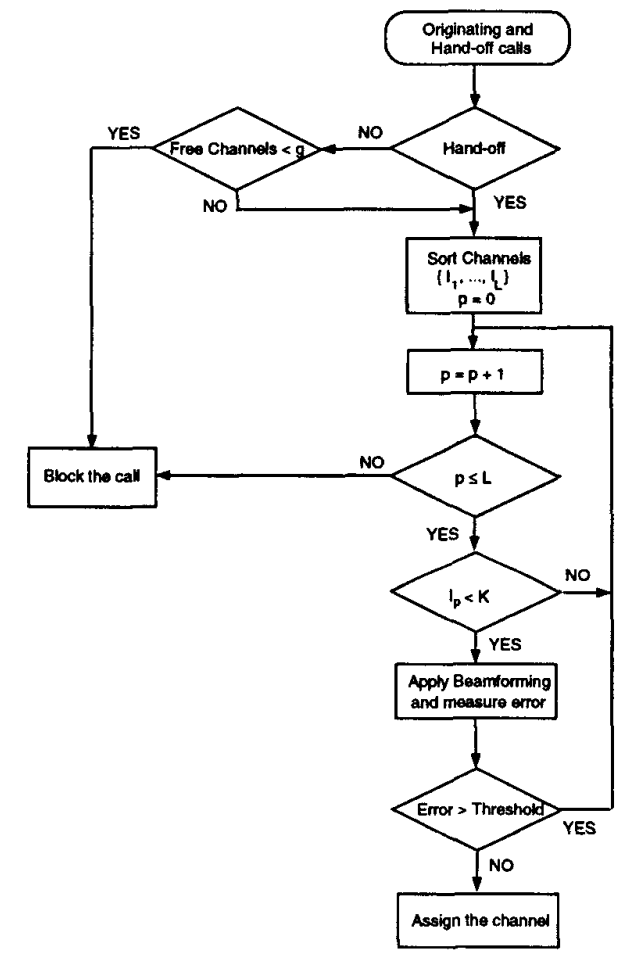

Figure 2: Flow Chart of the channel assignment algorithm in a system with antenna arrays.

The success probability $p_{i \mid i-1, M}$ is the probability that the ith transmitter can be captured successfully by a separate antenna beam when there are $M$ antenna elements. In the event of successful capture of ith transmitter, that user will be sharing the same channel with $(i-1)$ previously captured users. In that case all $i$ users will be communicating with the BS using the same physical channel. Monte Carlo simulation results for $p_{2 \mid 1, M}$ and $p_{3 \mid 2, M}$, success probabilities for 2-beam and 3 -beam adaptive arrays, are shown in Figures 3 and 4 .

Since there are $L$ distinct physical channels in the sys-

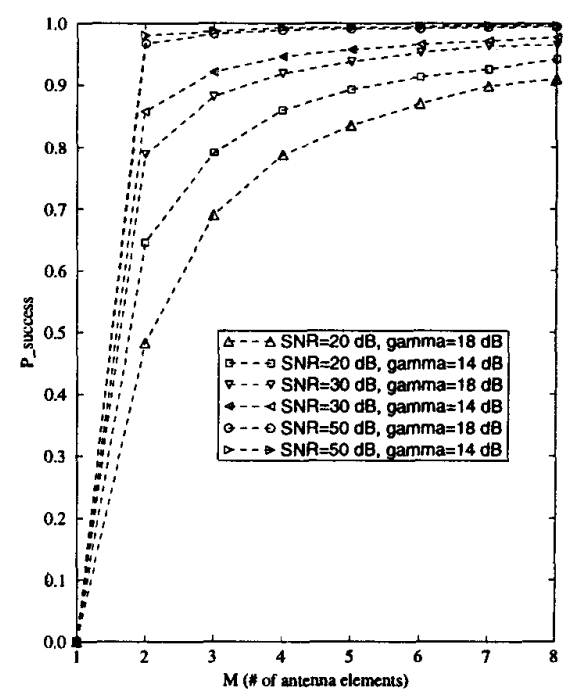

Figure 3: Success probability $p_{2 \mid 1, M}$ for a 2-beam adaptive

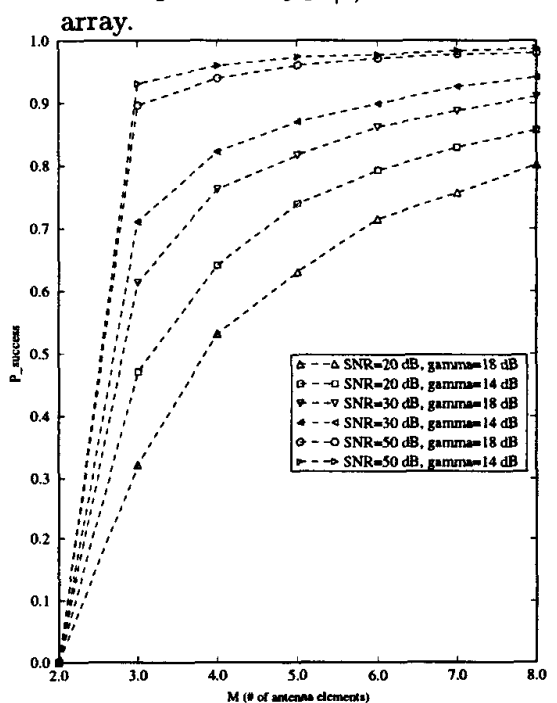

Figure 4: Success probability $p_{3 \mid 2, M}$ for a 3-beam adaptive array.

tem and each channel may be reused up to $K$ times we shall define $q_{n+1 \mid n}$, probability of successful reception of the $(n+1) t h$ user into the system when there are already $n$ users in the system

$$
q_{n+1 \mid n}=q_{N_{t+1} \mid N_{t}}\left(N_{t+1}=n+1 \mid N_{t}=n\right)
$$

where $N_{t}$ is the number of users in the system at time $t$ before a new call (user) arrives into the system ( $t$ is the time index which increases by one at each epoch corresponding to a new handoff or originating call). For a $K$-beam adaptive array system, we have shown [2] that success probabilities $q_{n+1 \mid n}$ are

$$
\begin{array}{r}
q_{n+1 \mid n}=\sum_{\substack{\left\{x_{2} \cdots x_{K} \mid x_{K} \leq \cdots \leq x_{2}\right\} \\
\times p\left[\left(x_{2}, \cdots, x_{K}\right) \mid x_{2}+\cdots+x_{K}=n-L\right]}} \hat{q}\left(n+1, x_{2}, \cdots, x_{K}\right)(9) \\
\text { where } \hat{q}\left(n+1, x_{2}, \cdots, x_{K}\right)=1-\left(1-p_{2 \nmid 1, M}\right)^{\left(L-x_{2}\right)} \times
\end{array}
$$


$\left(1-p_{3 \mid 2, M}\right)^{\left(x_{2}-x_{3}\right)} \times \cdots \times\left(1-p_{K \mid K-1, M}\right)^{\left(x_{K-1}-x_{K}\right)}$, and $\left(x_{2}, x_{3}, \cdots, x_{K}\right)$ denotes the vector representing the number of calls assigned to the channels in the second beam up to the $K$ th beam. Let $\alpha=\lambda+\gamma, \mu=$ $\eta+\nu, a=\frac{\alpha}{\mu}, b=\frac{\gamma}{\mu}, c=\frac{\lambda}{\mu}$, where $\gamma$ and $\eta$ are arrival rate and service rate for originating calls, $\lambda$ and $\nu$ are arrival rate and service rate for hand off calls, respectively. Figure 5 illustrates the state transition diagram of Markov chain model of the ongoing calls in a cell, where a $K$-beam adaptive array is used at the base station.

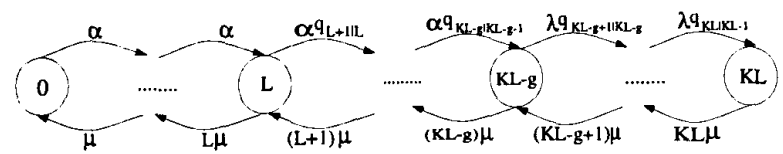

Figure 5: Queuing model of a cell with adaptive array under guard channel traffic policy.

From the network model in Figure 5, the state probabilities, $P(n) n=0,1, \cdots, K L$ (where $n$ is the number of ongoing calls in the cell) are derived, using queuing theory results [8]. It can be shown that the blocking probability $B_{O}$, for originating calls, and handoff calls blocking probability $B_{H}$ are

$$
\begin{aligned}
B_{O}= & {\left[\sum_{n=L}^{K L-g-1}\left(1-q_{n+1 \mid n}\right) \frac{a^{n}}{n !} \prod_{j=0}^{n-1} q_{j+1 \mid j}\right.} \\
& \left.+a^{K L-g} \sum_{n=K L-g}^{K L} \frac{c^{n-(K L-g)}}{n !} \prod_{j=0}^{n-1} q_{j+1 \mid j}\right] P(0) \\
B_{H}= & {\left[\sum_{n=L}^{K L-g-1}\left(1-q_{n+1 \mid n}\right) \frac{a^{n}}{n !} \prod_{j=0}^{n-1} q_{j+1 \mid j}\right.} \\
& +\sum_{n=K L-g}^{K L-1}\left(1-q_{n+1 \mid n}\right) \frac{a^{K L-g} c^{n-(K L-g)}}{n !} \\
& \left.\times \prod_{j=0}^{n-1} q_{j+1 \mid j}+\frac{a^{K L-g} c^{g}}{(K L) !} \prod_{j=0}^{K L-1} q_{j+1 \mid j}\right] P(0)
\end{aligned}
$$

We investigate another traffic policy that further decreases the blocking probability for handoff calls at the expense of slightly increasing the blocking probability for originating calls [7]. We assume that infinite queues are allowed for handoff calls. In a network where infinite queues are allowed for handoff calls, call blocking probabilities $B_{O}$ and $B_{H}$ are again computed and it is shown that the probability that a handoff call is delayed (due to queuing) is

$$
P_{H}(>0)=\frac{a^{K L-g} c^{g}}{(K L-1) !(K L-c)} \prod_{j=0}^{K L-1} q_{j+1 \mid j} P(0)
$$
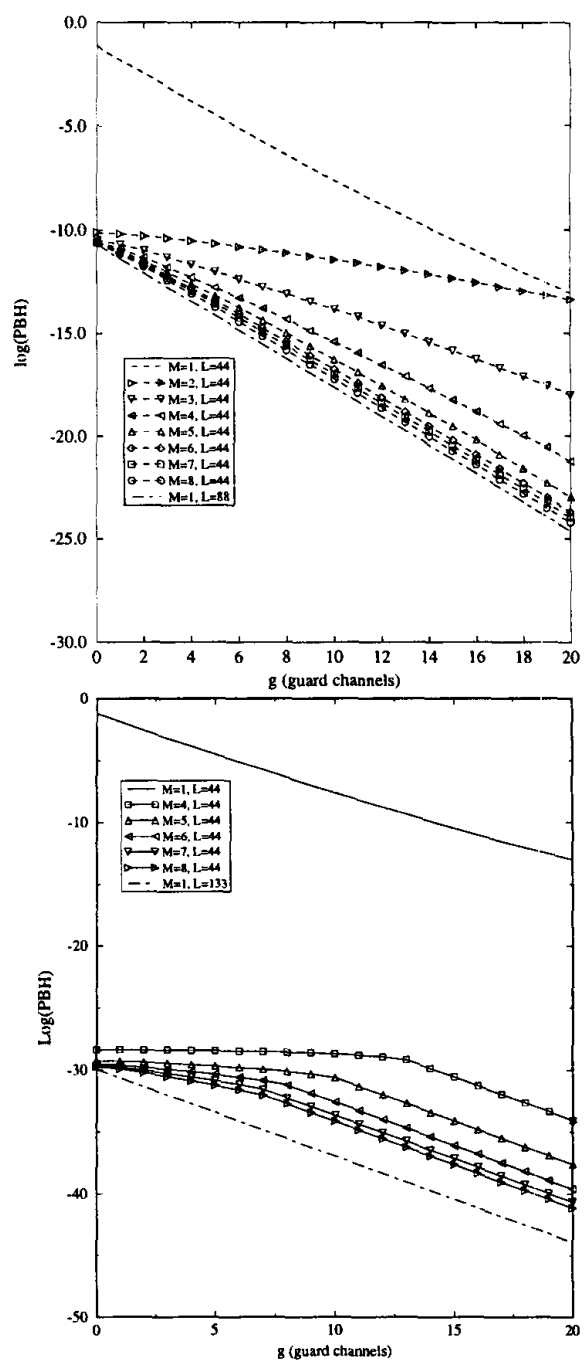

Figure 6: Blocking probabilities for hand off calls, $n_{H}$, for different number of antenna elements $M$ with $S N R=20 \mathrm{~dB}, \gamma=18 d B, \beta=4, a=40$, and $c=8$ Erlangs in a 2-beam (top) and 3-beam adaptive array system.

Also the probability that a hand off call waits more than a certain time $t$ is

$$
\begin{aligned}
P_{H}(>t) & =\frac{a^{K L-g} c^{g}}{(K L-1) !(K L-c)} e^{-\mu t(K L-c)} \\
& \prod_{j=0}^{K L-1} q_{j+1 \mid j} P(0) \\
& =e^{-\mu t(K L-c)} \times P_{H}(>0)
\end{aligned}
$$

From (13) the average delay $W_{H}$ for handoff calls is

$$
W_{H}=\frac{1}{\mu(K L-c)} \times P_{H}(>0),
$$

and the average delay for handoff calls that actually do experience a delay is

$$
D_{H}=\frac{1}{\mu(K L-c)}
$$




\section{Numerical Results}

In this section we present the numerical results to show the effectiveness of the network with adaptive arrays in terms of the traffic improvements in a cellular wireless network. We evaluate the performance of the system with parameters drawn from $[2,7]$. We choose a cell with $L=44$ channels with a total offered traffic $a=40$ Erlangs (heavy traffic), and a handoff traffic $c=8$ Erlangs. Blocking probabilities for handoff and originating calls in a 2-beam and 3-beam adaptive array system for different number of antenna elements $M$ are plotted in Figure 6. In these simulations, path loss exponent $\beta$ is assumed to be 4. The dropping rate of the blocking probabilities for handoff calls by increasing the number of antenna elements is emphasized in Figure 7.

The network with adaptive array can effectively handle higher offered traffic intensities $a$, compared to the network with single omni-directional antenna. The total carried traffic (in Erlangs) in the system is

$$
C=(a-c)\left(1-B_{O}\right)+c\left(1-B_{H}\right)
$$

Figure 8 illustrates the effect of adaptive arrays on the total carried traffic in the system.

\section{Conclusions}

A novel approach for traffic analysis of cellular wireless networks with beamforming capabilities at the base station is introduced. In our approach, the overall queuing model of a cell is derived by jointly considering the effects of the protocol at the network layer along with the effects of the signal processing at the physical layer directly on the actual cell model. Specifically, in this paper, the effects of the digital beamforming at the physical layer, along with GCT policy at the network layer are considered jointly on the overall queuing model of the cell and its traffic characteristics. Analytical as well as numerical results confirm the effectiveness of adaptive arrays in reducing the blocking probability of the calls and increasing the total carried traffic in the system.

\section{References}

[1] D. Everitt, "Traffic Engineering of the Radio Interface for Cellular Mobile Networks", IEEE Proc., pp. 13711382, Sept. 1994.

[2] Javad Razavilar, "Signal Processing and Performance Analysis for Optimal Resource Allocation in Wireless Networks," Ph.D. dissertation, Electrical Engineering Dept., University of Maryland, College Park, December 1998.

[3] Jack H. Winters, "Smart antennas for wireless systems," IEEE Personal Comm. Mag., pp. 23-27, Feb. 1998.
[4] J. Ward, R. T. Compton, "High Throughput Slotted ALOHA Packet Radio Networks with Adaptive Arrays," IEEE Trans. Comm., pp. 460-470, March 1993

[5] R. A. Monzingo and T. W. Miller, Introduction to Adaptive Arrays, Wiley, New York, 1980.

[6] S. Haykin, :Adaptive Filter Theory", Prentice Hall, Englewood cliffs, 1991.

[7] R. Guerin, "Queuing-Blocking System With Two Arrivals Stream and Guard Channels", IEEE Trans. Comm., Feb. 1988, pp 153-163.

[8] L. Kleinrock, Queuing Systems, vol I., 1985.

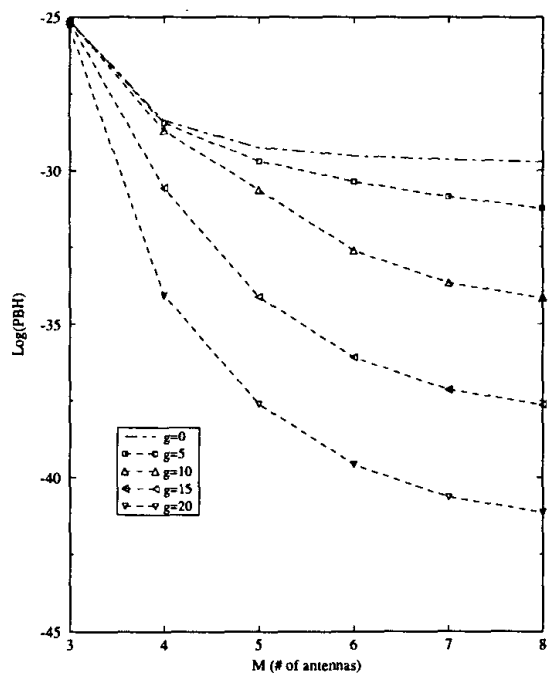

Figure 7: Blocking probabilities $B_{H}$ versus number of antenna elements $M$, with $S N R=20 \mathrm{~dB}, \gamma=$ $18 \mathrm{~dB}, a=40$, and $c=8$ Erlangs.

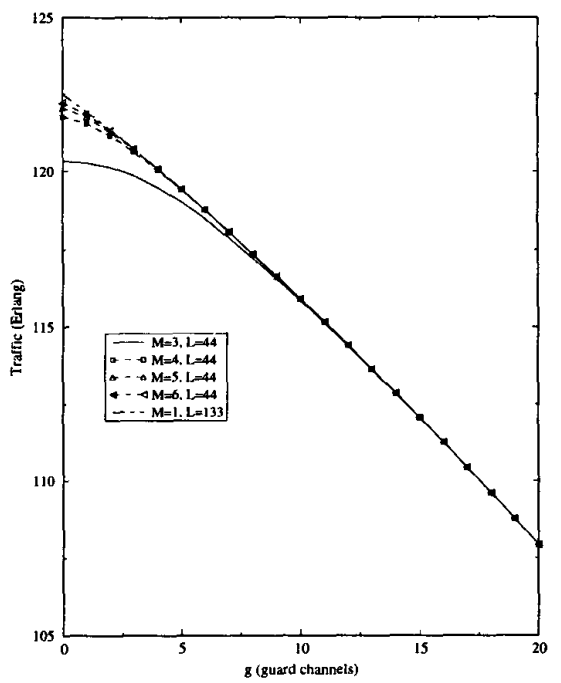

Figure 8: Total carried traffic $C$ for different number of antenna elements $M$ with $S N R=20 \mathrm{~dB}, \gamma=$ $18 \mathrm{~dB}, a=130$, and $c=8$ Erlangs. 